\title{
Effects of different bone marrow stimulation techniques on avascular zone meniscal defects
}

\author{
Bostan $\mathrm{B}^{1}$, Gevrek $\mathrm{F}^{2}$, Balta $\mathrm{O}^{3}$, Aytekin $\mathrm{K}^{4}$, Asci Mㄹ \\ Departments of Orthopaedics and Traumatology \& Anatomy, University of Giresun, School of Medicine, \\ Giresun, Turkey. kursadaytekin@gmail.com
}

\begin{abstract}
OBJECTIVES: In this study, we sought to investigate the effect of different amounts of Bone Marrow-Derived Mesenchymal Stem Cells (BMSCs), obtained by different BMSCs, on the healing of avascular zone meniscal defects. BACKGROUND: Treating avascular zone meniscal injuries has gained popularity. BMSCs contribute to the healing of avascular zone meniscal defects. The amount of BMSCs derived from different bone marrow stimulation techniques (BMSTs) varies, which could affect the therapeutic efficacy of this treatment.

METHODS: Fifty-four skeletally mature female New Zealand White rabbits were used after local ethical committee approval. A full thickness, $1.5 \mathrm{~mm}$ diameter defect was produced in the inner two-thirds of the anterior portion of the medial meniscus avascular zone using a biopsy punch. Animals were enrolled into three different groups according to BMST $(0.8 \mathrm{~mm}, 1.5 \mathrm{~mm}$, and $4 \mathrm{~mm})$. Medial menisci were harvested and prepared for histomorphometric, histologic and immune-histologic analyses.

RESULTS: Larger bridging tissues across the defect were detected in the 1.5- $\mathrm{mm}$ and 4-mm groups at 4 weeks $(p<0.05)$. The best quality score at the 1-,4- and 12-week endpoints was in $0.8 \mathrm{~mm}, 4 \mathrm{~mm}$ and $0.8 \mathrm{~mm}, 1.5$ $\mathrm{mm}$, respectively $(p<0.05)$. Immunostaining of experimental and control knees at the 1 -week endpoint revealed diffuse staining within the bridging tissue for PDGA, TGF $\beta-3$, BMP-2 and IGF. HSCORE for these pro-regenerative cytokines did not reach statistical significance at the 1-week endpoint $(p>0.05)$

CONCLUSION: The largest amount of BMSCs did not correlate with best quality and largest quantity of bridging tissue at the avascular zone in meniscal defects (Tab. 3, Fig. 4, Ref. 30).

KEY WORDS: mesenchymal stem cells, meniscus, avascular zone, microfracture, New Zealand White rabbit.
\end{abstract}

\section{Introduction}

Menisci have critical roles with respect to load bearing, transmission, lubrication and nutrition of articular cartilage (1). Peak contact stress, which is related to the degree of meniscectomy, is the major contributing factor to the development of osteoarthritis in the tibiofemoral joint. Therefore, in treating meniscal injuries, the preservation of as much meniscal tissue as possible is critical for preventing further degeneration (2). Meniscectomy significantly increases the risk of osteoarthritis (3). As a result, treating avascular zone meniscal injuries has gained popularity. Bone mar-

${ }^{1}$ Medical Palace Hospital, Department of Orthopaedics and Traumatology, Kayseri, Turkey, ${ }^{2}$ Gaziosmanpasa University, School of Medicine Department of Histology and Embriology, Tokat, Turkey, ${ }^{3}$ Gaziosmanpasa University, School of Medicine Department of Orthopaedics and Traumatology, Tokat, Turkey, ${ }^{4}$ Giresun University, School of Medicine Department of Orthopaedics and Traumatology, Giresun, Turkey, and ${ }^{5}$ Gaziosmanpasa University, School of Medicine Department of Biostatistics, Tokat, Turkey

Address for correspondence: $\mathrm{K}$. Aytekin, MD, PhD, Departments of Orthopaedics and Traumatology \& Anatomy, University of Giresun, School of Medicine, Gazi Caddesi, Debboy Mevkii, Gazipasa Kampusu, 28100 Giresun, Turkey.

Phone: +90.505.3737599, Fax: +90.454.3101696

Acknowledgement: The study was funded by the Gaziosmanpaşa University Scientific Research Projects Programme (51879863-33). row stem cells (4), growth factors (5), exogenous fibrin clots (6), trephination (7), stem cell therapy (8) and platelet rich plasma (9) have all been used in an effort to protect meniscal tissue. Driscoll et al (4) showed that bone marrow-derived mesenchymal stem cells (BMSCs) contribute to the healing of avascular zone meniscal defects. Min BH et al (10) quantified the amount of BMSCs derived from different bone marrow stimulation techniques (BMSTs) and suggested that the amount of BMSCs could affect the therapeutic efficacy of this treatment. However, we could not identify any studies investigating this effect.

In this study, we sought to investigate the effect of different amounts of BMSCs, obtained by different BMSTs, on the healing of avascular zone meniscal defects (AZMDs).

\section{Materials and methods}

The experimental protocols were approved by the local Institutional Animal Care and Use Committee. Fifty-four skeletally mature female New Zealand White rabbits, weighing an average of $3.5 \mathrm{~kg}$, were enrolled and divided into three equal groups. Preoperative analgesics and antibiotic prohylaxis were administered intramuscularly. The animals were intramuscularly anaesthetized with $10 \mathrm{mg} / \mathrm{kg}$ ketamin and $8 \mathrm{mg} / \mathrm{kg}$ xylazine. In each animal, both knees were operated on at the same time. Left knees were assigned to the control group and right knees were assigned to the treatment 


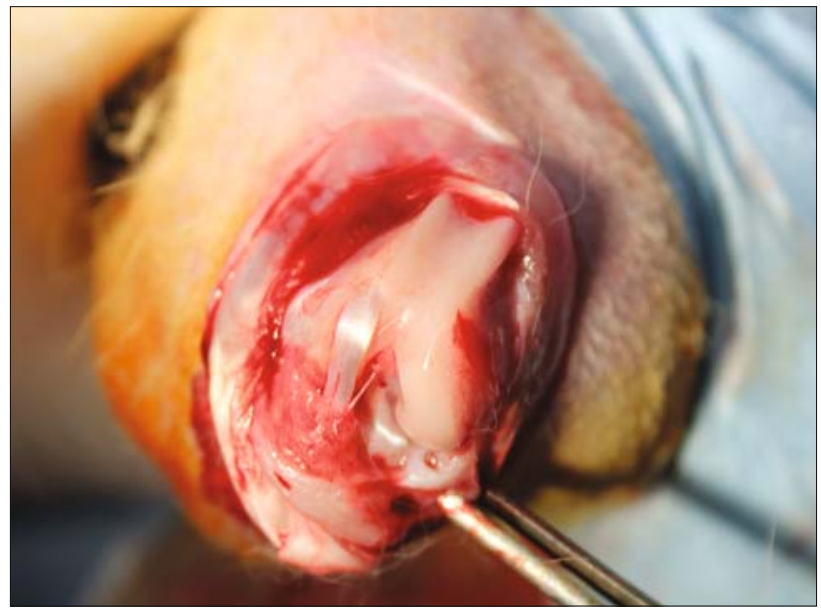

Fig. 1. An intraoperative view showing the defect in the avascular zone of the medial meniscus.

group. The knees were accessed through a medial parapatellar arthrotomy. The patella was laterally dislocated and the meniscus was exposed with maximal flexion. A full thickness, $1.5 \mathrm{~mm}$ diameter defect was produced in the inner two-thirds of the anterior portion of the medial meniscus avascular zone using a biopsy punch (Miltex,York,PA) (Fig. 1). This procedure was performed in both knees of each animal. The animals were enrolled into three different groups, according to BMST. BMSTs were modified according to the technique described by Min BH et al (10).

Kirschner wires of $0.8 \mathrm{~mm}, 1.5 \mathrm{~mm}$, and $4 \mathrm{~mm}$ diameters were used to make a microfracture for spilling different amounts of BMSCs. Microfractures were performed on the trochlear grove to a depth of three millimetres. Three consecutive drillings three millimetres apart from one another were performed in the $0.8 \mathrm{~mm}$ and $1.5 \mathrm{~mm}$ groups, whereas only one drilling was performed in the $4 \mathrm{~mm}$ group. The capsule and skin were closed in layers with absorbable sutures. Rabbits were maintained in their cages without movement restriction. A total of 54 rabbits were killed at the 1-, $4-$, and 12 -week endpoints ( $\mathrm{n}=54$ total; $\mathrm{n}=18$ in each group and $\mathrm{n}=6$ at three different time endpoints within each group). Medial menisci were harvested and prepared for histomorphometric, histologic and immunhistologic analyses.

\section{Tissue preparation}

After meniscus tissue specimens were grossly inspected and photographed, they were immediately fixed in $4 \%$ neutral buffered paraformaldehyde, decalcified in a fixative-added decalcification solution containing a fixative and ethylenediamine- tetraacetic acid, washed under running tap water, and then dehydrated through an ethanol dilution series and embedded in paraffin. Each specimen was then cut into 5-mm thick sections with a rotary microtome (Leica RM2125RT, China) in the radial plane, through the centre of the original defect. For histologic and histomorphometric analyses, the sections were stained with Safranin-O/Fast Green. The quantity and quality of regenerated meniscal tissues were evaluated as described below.

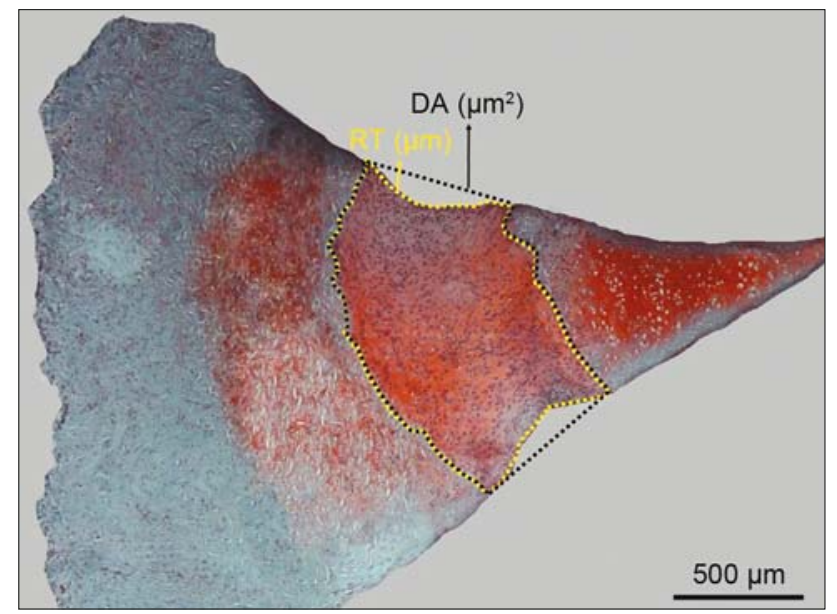

Fig. 2. A representative image of the original defect area and reparative bridging meniscal tissue at 4 weeks after injury. Using radial sections taken through the central portion of the defect, the area of the entire defect (black dotted line, DA) and the area of the reparative tissue (yellow dotted line, RT) were measured via a Niss element program installed research microscope. The reparative tissue area/defect area ratio (RT/DA) was used to quantify the amount of meniscal tissue bridging the defect in each meniscal tissue. Complete full-thickness bridging would result in a value of $100 \%(=1)$ and incomplete or partial-thickness bridging would result in a value less than $100 \%$ ( = 0-1)(Safranin-O, Scale bar: $500 \mu \mathrm{m})$.

\section{Tissue quantity analysis}

The quantity of reparative tissue bridging the meniscal defects was evaluated using 9-10 random serial tissue sections obtained from the central portion of each defect. To define the original defect, horizontal lines were drawn, connecting the superior and inferior surfaces of the meniscus at the borders of the $0.8-\mathrm{mm}$, $1.5-\mathrm{mm}$ and $4-\mathrm{mm}$ biopsies. Tissue regeneration was evaluated within the region bounded by these lines and within the adjacent native meniscal tissues. The area of the original defect (OD) and the area occupied by reparative tissue inside the defect (RT) were calculated using a Niss element digital image software system integrated into a computerized research light microscope (Nicon Eclipse E200, Japan). The quantity of reparative tissues was then expressed as the ratio of reparative tissue area to the entire defect area (RT/OD). Complete and full thickness bridging of the menis-

Tab. 1. Histologic tissue quality score (11).

\begin{tabular}{lc}
\hline Category & Points \\
\hline Reparative tissues with bonding & 2 \\
Bilateral bonds with surrounding meniscus & 1 \\
Partiall bonds with surrounding meniscus & 0 \\
No bond with surrounding meniscus & 2 \\
\hline Existence of fibrochondrocytes & 1 \\
Fibrochondrocytes exist diffusely in the reparative tissues & 0 \\
Fibrochondrocytes are localized in the reparative tissues & \\
No fibrochondrocytes in the reparative tissues & 2 \\
\hline Staining with safranin-O & 1 \\
Densely stained with safranin-O & 0 \\
Faintly stained with safranin-O & \\
Not stained with safranin-O & \\
\hline
\end{tabular}




\section{$630-635$}

cal defect would result in a value of $100 \%$, and incomplete bridging would result in a value less than $100 \%$ (4) (Fig. 2).

\section{Tissue quality analysis}

The evaluation of 3 dimensions of reparative meniscal tissue quality was performed using an established quantitative scoring system, as described in Table 1. Sections were stained at neutral $\mathrm{pH}$ with Safranin-O, which identifies areas rich in proteoglycans. Histologic scoring was performed by one investigator (FG), who was blinded to the treatment category $(4,11)$.

\section{Immunohistochemistry}

Radial meniscal tissue sections from the experimental and control knees of each rabbit across all groups were also immunohistochemically analysed to test for the presence of growth factors involved in meniscal cell proliferation and extracellular matrix synthesis, including transforming growth factor (TGF) $\beta$-3, insulin-like growth factor I (IGF-1),platelet-derived growth factor (PDGF)-A and bone morphogenetic protein (BMP)-2. It is postulated that growth factor quantities peak at approximately 1 week after injury, but we studied specimens from all of the time endpoints. Five micrometre thick unstained sections from $4 \%$ neutral buffered formalin fixed and paraffin embedded meniscal tissues were deparaffinized, hydrated, and kept in a citric acid solution for 5 minutes in a microwave (Nitellowawe MD 554 Arçelik) to antigen retrieval except for that of BMP-2 and were then immersed in $3 \%$ hydrogen peroxide to quench endogenous peroxidase activity. Next, slides were washed in phosphate-buffered saline (PBS) and incubated in $1.5 \%$ blocking serum for 1 hour to block nonspecific staining. Specimens were then incubated overnight at $4{ }^{\circ} \mathrm{C}$ in a moist environment and in the dark with the following primary antibodies: TGF $\beta-3$ (1:100 dilution; Santa Cruz Biotechnology, Santa Cruz, CA), IGF-1 (1:100 dilution; Santa Cruz Biotechnology), BMP-2
(1:200 dilution; Santa Cruz Biotechnology), and PDGF-A (1:200 dilution; Santa Cruz Biotechnology). Next, sections were washed with PBS, incubated with a biotinylated secondary antibody (Santa Cruz Biotechnology) for 30 minutes, and washed again with PBS. A streptavidin-marked secondary antibody was added to the tissue sample, incubated for 30 minutes in a humidified atmosphere in the dark, and washed three times with PBS. Finally, samples were incubated with AEC chromogen for 5-10 minutes to visualize the immunoreactivity, washed in distilled water, counterstained with haematoxylin, and then mounted with an aqueous mounting solution (Invitrogen). For the negative control, PBS was dropped onto the sections instead of a primary antibody, and the same procedures were applied as in the other steps. No immunostaining was observed in the negative control.

\section{Immunohistochemical evaluation}

All meniscal tissue sections were viewed under a microscope to identify the immune reactive cells. A semi-quantitative evaluation system, a scoring scale, was used based on the severity of staining that occurred as a result of the antigen-antibody reaction. In this scale, a total of four values were used, graded from one negative to three positive. The interpretations of the grades are as follows: (-): no staining, $(+)$ : weakly detectable staining, $(++)$ : medium severity staining, and $(+++)$ : severe staining. Through using this scale, the immune staining of cartilage cells was evaluated.

\section{Semi-quantitative HSCORE Analysis}

Five areas were randomly selected from the sections on each animal and were to be examined under a light microscope with 40x magnification. The categorical enumeration of the cells within these areas was made according to their immune staining intensity. The average results of blind counts were taken. During these counts, both the number of the cells showing a positive immu-

Tab. 2. Reparative Tissue Area/Defect Area (RT/DA).

\begin{tabular}{|c|c|c|c|c|c|c|c|c|c|c|}
\hline \multirow{3}{*}{ Time } & \multicolumn{8}{|c|}{ Groups } & \multirow{3}{*}{$\mathrm{F}$} & \multirow{3}{*}{$\mathrm{p}$} \\
\hline & \multicolumn{2}{|r|}{ Control } & \multicolumn{2}{|r|}{$0.8 \mathrm{~mm}$} & \multicolumn{2}{|r|}{$1.5 \mathrm{~mm}$} & \multicolumn{2}{|r|}{$4 \mathrm{~mm}$} & & \\
\hline & $\mathrm{n}$ & Mean \pm SD & $\mathrm{n}$ & Mean \pm SD & $\mathrm{n}$ & Mean \pm SD & $\mathrm{n}$ & Mean \pm SD & & \\
\hline 1 week & 6 & $0.43 \pm 0.04^{(\mathrm{A})}$ & 6 & $0.44 \pm 0.1^{(\mathrm{A})}$ & 6 & $0.5 \pm 0.09^{(\mathrm{A})}$ & 6 & $0.47 \pm 0.1^{(\mathrm{A})}$ & 0.687 & 0.570 \\
\hline 4 week & 6 & $0.49 \pm 0.05^{(\mathrm{a}, \mathrm{A})}$ & 6 & $0.61 \pm 0.13^{(\mathrm{ab}, \mathrm{A})}$ & 6 & $0.69 \pm 0.07^{(\mathrm{b}, \mathrm{B})}$ & 6 & $0.74 \pm 0.02^{(\mathrm{b}, \mathrm{B})}$ & 11.792 & $<0.001$ \\
\hline 12 week & 6 & $0.91 \pm 0.03^{(\mathrm{B})}$ & 6 & $0.94 \pm 0.03^{(\mathrm{B})}$ & 6 & $0.93 \pm 0.05^{(\mathrm{C})}$ & 6 & $0.91 \pm 0.03^{(\mathrm{C})}$ & 0.887 & 0.465 \\
\hline $\mathrm{F}$ & & 243.543 & & 43.116 & & 52.254 & & 78.922 & & \\
\hline $\mathrm{p}$ & & $<0.001$ & & $<0.001$ & & $<0.001$ & & $<0.001$ & & \\
\hline
\end{tabular}

Uppercase superscripts represent differences of colons, and lowercase superscripts represent differences of lines, no significant differences between groups with the same superscripts, F: Analysis of variance (ANOVA), $\mathrm{p}<0.05$ represents statistical significance

Tab. 3. Tissue Quality Score (range 0-6).

\begin{tabular}{|c|c|c|c|c|c|c|}
\hline \multirow{3}{*}{ Time } & \multicolumn{4}{|c|}{ Groups } & \multirow{3}{*}{$\mathrm{F}$} & \multirow{3}{*}{$\mathrm{p}$} \\
\hline & Control & $0.8 \mathrm{~mm}$ & $1.5 \mathrm{~mm}$ & $4 \mathrm{~mm}$ & & \\
\hline & Mean \pm SD & Mean \pm SD & Mean \pm SD & Mean \pm SD & & \\
\hline 1 Weeks & $2.45 \pm 1.31^{(a, A)}$ & $5.6 \pm 0.81^{(b, A)}$ & $3.4 \pm 0.5^{(c, A)}$ & $3 \pm 2.49^{(a c, A)}$ & 33.148 & $<0.001$ \\
\hline 4 Weeks & $3.8 \pm 0.41^{(a, B)}$ & $4.4 \pm 1.99^{\text {(ас. в) }}$ & $2,8 \pm 1^{(b, B)}$ & $5 \pm 0^{(c, B)}$ & 23.207 & $<0.001$ \\
\hline 12Weeks & $4.8 \pm 0.41^{(a, C)}$ & $6 \pm 0^{(b, A)}$ & $6 \pm 0^{(b, C)}$ & $5 \pm 0^{(a, B)}$ & 297.250 & $<0.001$ \\
\hline $\mathrm{F}$ & 61.537 & 13.464 & 209.767 & 21.312 & & \\
\hline $\mathrm{p}$ & $<0.001$ & $<0.001$ & $<0.001$ & $<0.001$ & & \\
\hline
\end{tabular}

Uppercase superscripts represent differences of colons, and lowercase superscripts represent differences of lines, no significant differences between groups with the same superscripts, F: Analysis of variance (ANOVA), $\mathrm{p}<0.05$ represents statistical significance 

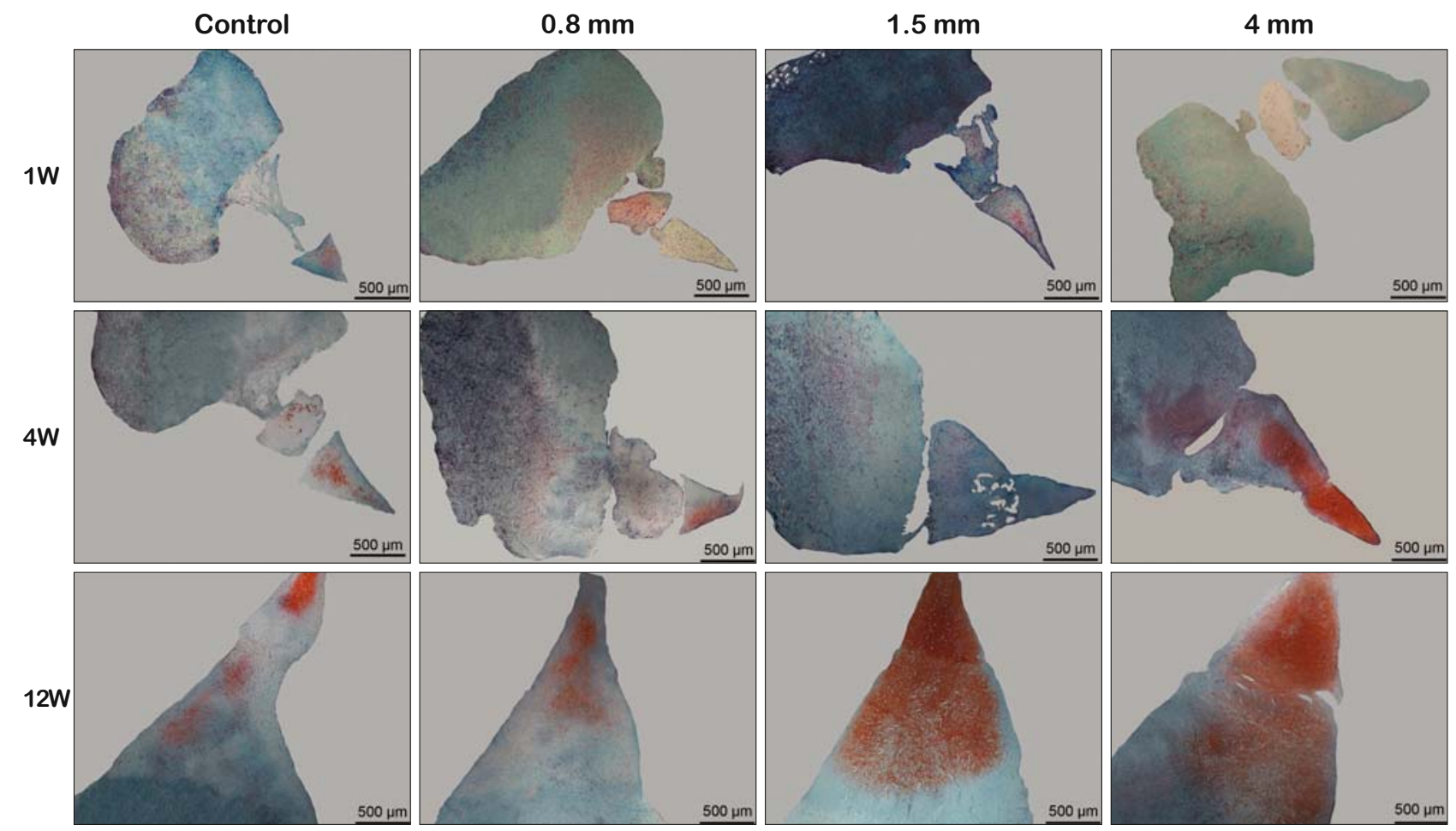

Fig. 3. Representative microscopic images of regenerated meniscus sections stained with Safranin-O at control, $0.8 \mathrm{~mm}, 1.5 \mathrm{~mm}$ and $4 \mathrm{~mm}$ BMST in each treatment week $(1,4$, and 12 weeks) after meniscus injury. Scale bar: $500 \mu \mathrm{m}$.

noreactivity and the immunoreactive intensity degrees of these cells were considered in all cells that were stained and not stained. For estimating the results of these counts, the HSCORE formula $\sum \mathrm{Pi}(\mathrm{i}+1)$ was used. In this formula, iis the staining intensity score and Piis the percentage of all cells that are stained $(12,13)$.

\section{Statistical analysis}

Descriptive analyses were performed to provide information on the general characteristics of the study population. The data from the variables were represented as the mean \pm standard deviation. A one-way ANOVA was used to compare the means of variables between groups. A $p<0.05$ was considered significant. Analyses were performed using commercial software (IBM SPSS Statistics 19, SPSS Inc., an IBM Co., Somers, NY).

\section{Results}

\section{Tissue quantity analysis}

The gross inspection of the meniscal tissue bridging the defects revealed a significant closure of the defects in the $1.5-\mathrm{mm}$ and 4-mm groups at 4 weeks compared to that of the control and $0.8-\mathrm{mm}$ groups. However, the gross inspections of the meniscal tissue bridging the defects at 1 and 12 weeks revealed that the closures of the defects were similar in all of the groups. A detailed microscopic analysis of tissues bridging the defects revealed significantly better scores in the $1.5-\mathrm{mm}$ and $4-\mathrm{mm}$ groups at 4 weeks $(p<0.05)$. However, there was no significant difference detected between all groups at 12 weeks (Tab. 2).

\section{Tissue quality analysis}

Better tissue quality scores were detected in the BMST $(0.8$ $\mathrm{mm}, 1.5 \mathrm{~mm}$, and $4 \mathrm{~mm}$ ) groups compared to the controls at different time endpoints. The best quality score at the 1,4 , and 12 week endpoints was in the $0.8-\mathrm{mm}, 4-\mathrm{mm}$ and $0.8-\mathrm{mm}$, and $1.5-\mathrm{mm}$ groups, respectively $(\mathrm{p}<0.05)$ (Tab. 3 and Fig. 3).

\section{Immunostaining}

Immunostaining of the experimental and control knees at 1 week revealed diffuse staining within the bridging tissue for PDGA, TGF $\beta-3$, BMP-2 and IGF. HSCORE for these proregenerative cytokines did not have statistical significance at the 1 week endpoint $(p>0.05)$. The highest scores of staining for TGF $\beta-3$, PDGA were detected in the 1.5-mm group at the 4-week endpoint, however, the highest BMP-2 and IGF scores were not significantly different in the control and experimental knees across all of the groups (Fig. 4).

\section{Discussion}

Since the menisci have important roles with regard to the protection of cartilage by various mechanisms, such as load bearing, transmission, lubrication and nutrition of articular cartilage, the preservation of as much menisci tissue as possible during surgery is an idea that has gained popularity in recent years (1). Isolated arthroscopic meniscal repair yields favourable long term clinical and functional results $(14,15)$. The arthroscopic repair of isolated meniscal tears revealed better long term clinical and radiological results compared to those of meniscectomies (16-18). However, 

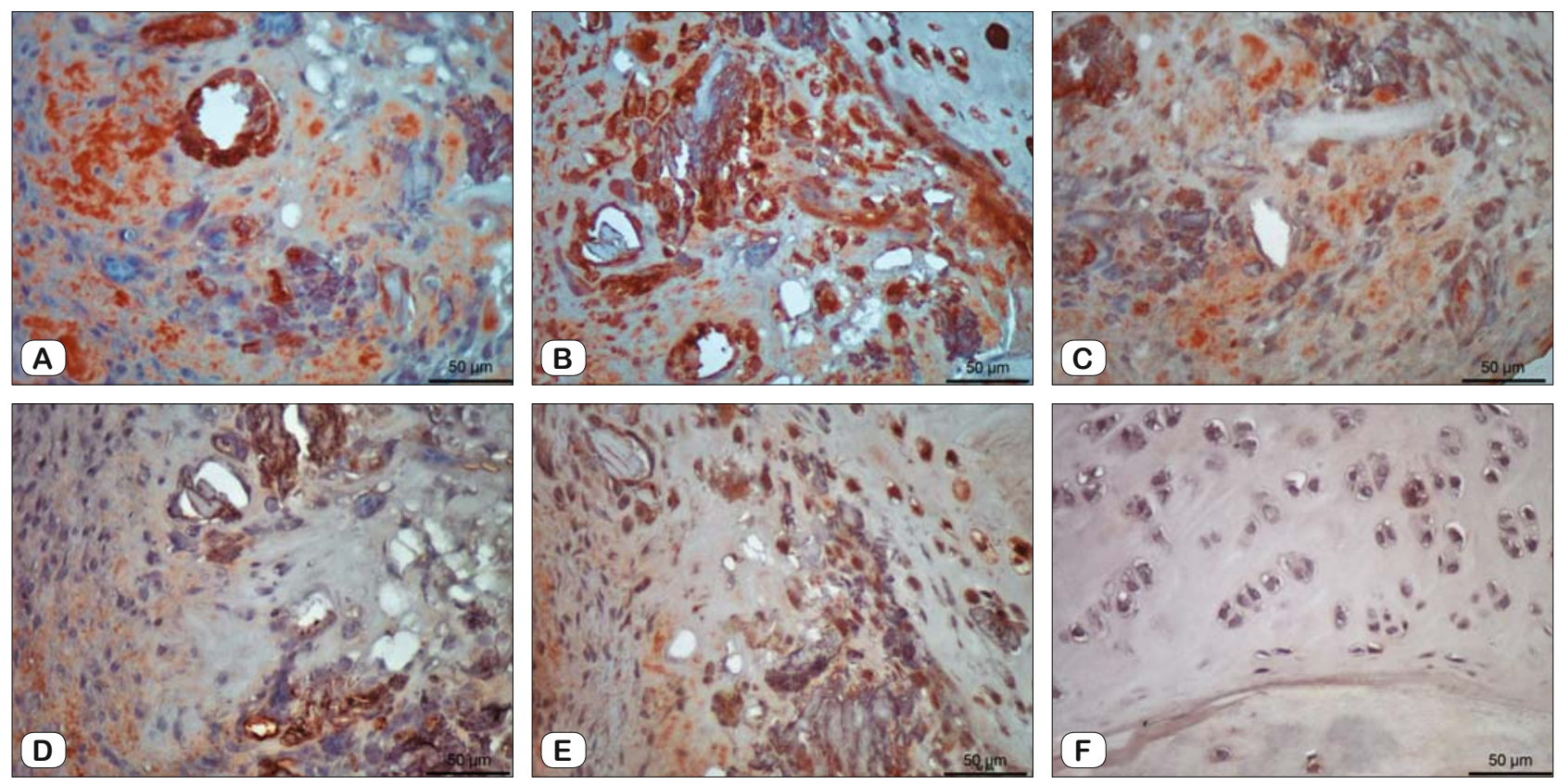

Fig. 4. Representative images of the immunohistochemical expression of proregenerative cytokines. In all of the BMST and control meniscal tissues, immunohistochemistry assays demonstrated positive staining for TGFb-3, PDGA, IGF, and BMP-2 within the reparative meniscal tissue in the $1.5 \mathrm{~mm}$ MS group at 4 weeks after injury. When staining for TGFb-3 and PDGA, the highest positive immune reactivity has been shown. A: TGFb-3, B: PDGA, C: IGF, D: BMP-2, E: Control, and F: Negative control. (IHC, Scale bar: $50 \mu \mathrm{m}$ ).

good results of the arthroscopic repair of red-red zone menisci do not allow us to predict the results of red-white or avascular zone repairs because the blood supply, which is crucial for healing, is limited or deficient in the central meniscus. Therefore, many studies, mostly experimental, have been conducted to investigate the effects that different augmentation techniques have on the repair of avascular zone meniscal injury or defects.

Long term clinical studies on the results of avascular zone meniscal repair are sparse. Rubman MH et al assessed the results of the arthroscopic repairs of 198 meniscal tears in the avascular zone, which had a mean follow up time of 42 months. A reoperation rate of $20 \%$ was detected. However, second look arthroscopy evaluation revealed that of the 91 repairs, 68 knees ( $74 \%$ ) were either not healed or only partially healed. In this study, although a tibiofemoral joint complaint was clinically detected in 39 knees, the rate of healing in repairs detected by arthroscopy was much less (19). Noyes FR et al assessed the results of 29 arthroscopic repairs of avascular zone meniscal tears; of these, 21 knees underwent concomitant ACL reconstruction. Of the 29 repairs, only 3 knees were re-operated on, which represents a high success rate (20). A high success rate may be attributable to mesenchymal stem cells rising from the femoral and tibial tunnels of ACL reconstruction. The success rate may be as high as $75 \%$ by clinical examination, which does not represent the rate detected by second look arthroscopy (21-23).

Zellner J et al studied the effects of PRP and BMP7, in combination with a hyaluronan collagen matrix, on the regeneration of avascular zone meniscal defects and found that although the procedure has a positive effect on meniscal healing, the improvement was not significant (5). Trephination and an exogenous fibrin clot were used in the past to augment repair $(6,7)$. Cook JL et al com- pared the effectivity of trephination and repair with a novel conduit composed of bioabsorbable poly-L-lactic acid for the augmentation of avascular zone meniscal tears and found the conduit treatment to be more effective in regard to tissue healing and biomechanical integrity (24). Locally applied vascular endothelial growth factor was investigated, and it was found to have no contribution to angiogenesis and thus no contribution to meniscal healing $(25,26)$.

Mesenchymal stem cells that are multipotent by nature are easily accessible from the bone marrow, periosteum, and adipose and synovial linings of major joints (27). A study by Horie $\mathrm{M}$ et al confirmed the efficacy of allogenic mesenchymal stem cells for improving meniscal defects (27). The intraarticular injection of synovial stem cells promotes a regeneration of massive meniscal defects (28). Bone marrow derived mesenchymal stem cells were harvested and cultured accordingly and applied to a meniscal repair of avascular zone meniscal tears and defects in two studies. The results of these studies confirmed the contribution of mesenchymal stem cells on the quality and quantity of the healing of defects and tears $(29,30)$. We used the rabbit meniscal defect model described by Driscoll MD et al (4), in which the authors used $2.4 \mathrm{~mm}$ pins to create a microfracture to detect a modest improvement of meniscal defects in the marrow stimulation group. The current work was inspired by the study of Min BH et al (10), in which they showed that different BMSTs mobilize different amounts of mesenchymal stem cells. They detected that the number of BMSCs drained was largest in the subchondral 4-mm opening group, followed by the 1.5-mm and $0.8-\mathrm{mm}$ microfracture groups, in that order (10). We investigated whether the amount of BMSCs affects the quality and quantity of the regeneration of meniscal defects. Therefore, we created different BMSTs according to the study of Min BH et al (10). 
The largest tissue quantity in the present study was detected in the 1.5-mm and 4-mm groups at 4 weeks. However, this measurement at the 1-and 12-week endpoints was not statistically significant in all of the groups, including the controls. Tissue quality scores were significantly better in all treatment groups compared to the controls at all endpoints. The best quality scores at the 1-,4-, and 12-week endpoints were in the $0.8-\mathrm{mm}, 4-\mathrm{mm}$ and 0.8 -mm groups, together with the 1.5-mm group, respectively. The best HSCORE for the immunostaining of TGF $\beta-3$, PDGA was detected in the $1.5 \mathrm{~mm}$ group at the 4-week endpoint. This study proved the efficacy of BMSCs on the healing of avascular zone meniscal defects. However, the largest amount of BMSCs, which stems from largest hole or from the most frequent number of holes, did not correlate with best quality and quantity of bridging tissue at the avascular zone meniscal defects.

The clinical relevance of this study could be of importance for the arthroscopic repair of avascular zone meniscal defects and cartilage injuries.

\section{References}

1. Makris EA, Hadidi P, Athanasiou KA. The knee meniscus: structurefunction, pathophysiology, current repair techniques, and prospects for regeneration. Biomaterials 2011; 32 (30): 7411-7431.

2. Lee SJ, Aadalen KJ, Malaviya P, Lorenz EP, Hayden,J K, Farr J, Cole B J. Tibiofemoral contact mechanics after serial medial meniscectomies in the human cadaveric knee. Am J Sports Med 2006; 34 (8): 1334-1344.

3. Roos H, Laurén M, Adalberth T, Roos EM, Jonsson K, Lohmander L. Knee osteoarthritis after meniscectomy: prevalence of radiographic changes after twenty-one years, compared with matched controls. Arthritis Rheum 1998; 41 (4): 687-693.

4. Driscoll MD, Robin BN, Horie M, Hubert ZT, Sampson HW, Jupiter D C, Reeve RE. Marrow stimulation improves meniscal healing at early endpoints in a rabbit meniscal injury model. Arthroscopy 2013; 29 (1): 113-121.

5. Zellner J, Taeger C, Schaffer M, Roldan JC, Loibl M, Mueller MB, Nerlich M. Are applied growth factors able to mimic the positive effects of mesenchymal stem cells on the regeneration of meniscus in the avascular zone? Biomed Res Int 2014; 537686.

6. Arnoczky SP, Warren RF, Spivak JM. Meniscal repair using an exogenous fibrin clot. An experimental study in dogs. J Bone Joint Surg Am 1988; 70 (8): 1209-1217.

7. Zhang Z, Arnold JA, Williams T, McCann B. Repairs by trephination and suturing of longitudinal injuries in the avascular area of the meniscus in goats. Am J Sports Med 1995; 23 (1): 35-41.

8. Murphy JM, Fink DJ, Hunziker EB, Barry FP. Stem cell therapy in a caprine model of osteoarthritis. Arthritis Rheum 2003; 48 (12): 3464-3474.

9. Delos D, Rodeo SA. Enhancing meniscal repair through biology: plateletrich plasma as an alternative strategy. Instructional Course Lectures 2010; 60: $453-460$.

10. Min BH, Choi WH, Lee YS, Park SR, Choi BH, Kim YJ, Yoon JH. Effect of different bone marrow stimulation techniques (BSTs) on MSCs mobilization. J Orthop Res 2013; 31 (11): 1814-1819.

11. Ishida K, Kuroda R, Miwa M, Tabata Y, Hokugo A, Kawamoto T, Kurosaka M. The regenerative effects of platelet-rich plasma on meniscal cells in vitro and its in vivo application with biodegradable gelatin hydrogel. Tissue Eng 2007; 13 (5): 1103-1112.

12. Cheon KW, Lee HS, Parhar IS, Kang IS. Expression of the second isoform of gonadotrophin-releasing hormone (GnRH-II) in human endometrium throughout the menstrual cycle. Mol Hum Reprod 2001; 7 (5): 447-452.
13. Godbole GB, Modi DN, Puri CP. Regulation of homeobox A10 expression in the primate endometrium by progesterone and embryonic stimuli. Reproduction 2007; 134 (3): 513-523.

14. Johnson MJ, Lucas GL, Dusek JK, Henning CE. Isolated arthroscopic meniscal repair: a long-term outcome study (more than 10 years). Am J Sports Med 1999; 27 (1): 44-49.

15. Majewski M, Stoll R, Widmer H, Müller W, Friederich NF. Midterm and long-term results after arthroscopic suture repair of isolated, longitudinal, vertical meniscal tears in stable knees. Am J Sports Med 2006; 34 (7): 1072-1076.

16. Paxton ES, Stock MV, Brophy RH. Meniscal repair versus partial meniscectomy: a systematic review comparing reoperation rates and clinical outcomes. Arthroscopy 2011; 27 (9): 1275-1288.

17. Lutz C, Dalmay F, Ehkirch FP, Cucurulo T, Laporte C, Le Henaff G, Seil R. Meniscectomy versus meniscal repair: 10years radiological and clinical results in vertical lesions in stable knee. Orthop Traumatol Surg Res 2015; 101 (8): S327-S331.

18. Stein T, Mehling AP, Welsch F, von Eisenhart-Rothe R, Jager A. Long term outcome after arthroscopic meniscal repair versus arthroscopic partial meniscectomy for traumatic meniscal tears. Am J Sports Med 2010; 38 (8): $1542-1548$.

19. Rubman MH, Noyes FR, Barber-Westin SD. Arthroscopic repair of meniscal tears that extend into the avascular zone a review of 198 single and complex tears. Am J Sports Med 1998; 26 (1): 87-95.

20. Noyes FR, Barber-Westin S D. Arthroscopic repair of meniscus tears extending into the avascular zone with or without ACL reconstruction in patients 40 years of age and older. Arthroscopy 2000; 16: 822 .

21. Gallacher PD, Gilbert RE, Kanes G, Roberts SN J, Rees D. White on white meniscal tears to fix or not to fix? Knee 2010; 17 (4): 270-273.

22. Noyes FR,Barber-Westin SD. Arthroscopic repair of meniscal tears extendingin to the avascular zone in patients younger than twenty years of age. Med Am J Sports 2002; 30 (4): 589-600.

23. Papachristou G, Efstathopoulos N, Plessas S, Levidiotis C, Chronopoulos E, Sourlas J. Isolated meniscal repair in the avascular area. Acta Orthop Bel 2003; 69 (4): 341-345.

24. Cook JL, Fox DB. A novel bioabsorbable conduit augments healing of avascular meniscal tears in a dog model. Am J Sports Med 2007; 35 (11): $1877-1887$.

25. Kopf S, Birkenfeld F, Becker R, Petersen W, Stärke C, Wruck CJ, Pufe T. Local treatment of meniscal lesions with vascular endothelial growth factor. J Bone Joint Surg Am 2010; 92 (16): 2682-2691.

26. Petersen W, Pufe T, Stärke C, Fuchs T, Kopf S, Neumann W, Becker R. The effect of locally applied vascular endothelial growth factor on meniscus healing: gross and histological findings. Arch Orthop Trauma Surg 2007; 127 (4): 235-240

27. Horie M, Driscoll MD, Sampson H W, Sekiya I, Caroom CT, Prockop DJ, Thomas DB. Implantation of allogenic synovial stemcells promotes meniscal regeneration in a rabbit meniscal defect model. J Bone JointSurg Am 2012; 94 (8): 701-712.

28. Hatsushika D, Muneta T, Horie M, Koga H, Tsuji K, Sekiya I. Intraarticular injection of synovial stemcells promotesmeniscal regeneration in a rabbit massive meniscal defect model. J Orthop Res 2013; 31 (9): 1354-1359.

29. Izuta Y, Ochi M, Adachi N, Deie M, Yamasaki T, Shinomiya R. Meniscal repair using bone marrow-derived mesenchymal stemcells: experimental study using green fluorescent protein transgenicrats. Knee 2005; 12 (3): 217-223. 30. Dutton AQ, Choong PF, Goh JC, Lee EH, Hui JHP. Enhancement of meniscal repair in the avascular zone using mesenchymal stemcells in a porcine model. J Bone Joint Surg Br 2010; 92 (1): 169-175.

Received June 10, 2018. Accepted July 17, 2018. 